\title{
Dynamics of coherently pumped lasers with linearly polarized pump and generated fields
}

\author{
R. Corbalán \\ Departament de Física, Universitat Autònoma de Barcelona, 08193 Bellaterra, Spain \\ R. Vilaseca \\ Departament de Física i Enginyeria Nuclear, ETSEIT, Universitat Politecnica de Catalunya, 08222 Terrassa, Spain \\ M. Arjona and J. Pujol \\ Departament d'Optica i Optometría, Universitat Politecnica de Catalunya, 08222 Terrassa, Spain \\ E. Roldán \\ Departament Interuniversitari d'Optica, Universitat de Vatencia, 46100 Burjassot, Spain \\ G. J. de Valcárcel \\ Departament de Física Aplicada, ETSII, Universitat Politecnica de Valencia, 46071 Valencia, Spain
}

(Received 19 October 1992; revised manuscript received 29 March 1993)

\begin{abstract}
The influence of light polarization on the dynamics of an optically pumped single-mode laser with a homogeneously broadened four-level medium is theoretically investigated in detail. Pump and laser fields with either parallel or crossed linear polarizations are considered, as are typical in far-infraredlaser experiments. Numerical simulations reveal dramatically different dynamic behaviors for these two polarization configurations. The analysis of the model equations allows us to find the physical origin of both behaviors. In particular, the crossed-polarization configuration is shown to be effective in decoupling the pump and laser fields, thus allowing for the appearance of Lorenz-type dynamics.
\end{abstract}

PACS number(s): 42.60.Mi, 42.50.Lc, 42.55. -f

\section{INTRODUCTION}

Extensive research over the last fifteen years has shown that lasers are among the most versatile physical systems to study a variety of temporal phenomena characteristic of nonlinear dissipative systems such as, for example, the onset of spontaneous pulsations in the output intensity that may be regular, quasiperiodic, or chaotic [1]. In particular, optically pumped molecular lasers (OPML) in addition to their practical interest as a source of stable coherent radiation in different regions of mid- and farinfrared [2], are very interesting systems from the viewpoint of nonlinear dynamics. In fact, OPML have allowed the experimental realization of a rich variety of dynamical behaviors that include (i) full amplitude periodic spiking associated with sustained relaxation oscillations, directly from the onset of laser threshold $[3,4]$; (ii) high-frequency periodic pulsing at higher pump powers, associated with pump-induced Rabi sideband oscillation [4]; (iii) a behavior remarkably similar [5-10] to the predictions of the paradigmatic Lorenz-Haken model for a plane-wave single-mode homogeneously broadened two-level laser [11-13]; and (iv) transitions to chaos by type-I [14] and type-III [15] intermittencies.

From the theoretical point of view OPML have been extensively studied in the framework of single-mode three-level models [16-21] with the aim to capture the $a$ priori most salient feature of these lasers, namely, the coherent interaction of pump and lasing emission fields, which distinguishes them from ordinary incoherently pumped two-level lasers and can lead to characteristic dynamic behavior as discussed by Harrison and coworkers $[19,20]$. Indeed, for specific domains of parameter values three-level laser models with either homogeneous broadening [4] or inhomogeneous (Doppler) broadening [22-26] have shown reasonably good qualitative agreement [4] and even a surprising similarity [22-26] with corresponding experimental findings. These coincidences suggest that these models take into account physical factors playing an important role in OPML dynamics. However, at this moment they can be only considered as a first step towards the understanding of the behavior of OPML since they do not take into account additional physical factors that are also present in the real laser, namely, longitudinal and transverse spatial dependence of pump and laser fields, $M$-level degeneracy of the radiatively coupled levels, and polarizations of the two laser fields. In principle any of these factors could have an influence in the laser dynamics that is worth investigating.

To take into account simultaneously all the physical factors above would represent a formidable task practically unaffordable with commonly available computers. In this paper we analyze the influence of light polarization and level degeneracy using a simple homogeneously broadened four-level OPML model.

One can expect to find a clear influence of these factors based on several previous experimental observations. The possibility of pump-intensity-driven polarization control has been demonstrated by Petukov, Tochitsky, 
and Churakov in an optically pumped $\mathrm{CO}_{2}$ laser oscillating in the $4.3-\mu \mathrm{m}$ region with a polarization-insensitive cavity [27]. Weiss and co-workers observed on the 81.5$\mu \mathrm{m}[6-8]$ and the $153-\mu \mathrm{m}[7,10]$ lines of the $\mathrm{NH}_{3}$ laser, whose linear polarization was orthogonal to the pump field polarization, a dynamic behavior remarkably different from that of the $374-\mu \mathrm{m} \mathrm{NH}_{3}$ line $[28,29]$ whose polarization was parallel to that of the pump field. In order to mimic the experimental conditions of Weiss and co-workers, pump and laser fields with fixed linear polarizations are considered through this paper. The interesting case of an OPML with a polarization-insensitive cavity in which the polarization state of the laser light can change in addition to the amplitude and phase changes that are usually investigated remains to be addressed in a future work. Very recently Bielawski, Derozier, and Glorieux [30] have shown theoretically and experimentally that the laser light polarization also plays an important role on the dynamics of a class of lasers different from the OPML considered here. They studied an Nd-doped optical fiber laser which is an incoherently pumped class-B laser.

The paper is organized as follows. In Sec. II we refer to a homogeneously broadened four-level model proposed previously by some of us [31], which allows us to consider arbitrary polarizations for pump and laser fields and the effect of level degeneracy in the simplest configuration: level scheme $J_{a}=0 \rightarrow J_{b}=1 \rightarrow J_{c}=0$ (see Fig. 1). Here we study this model in detail both analytically and numerically for the case of linear polarizations for pump and laser fields and compare its predictions with those of twoand three-level models. The case of crossed polarizations is studied in Sec. III. For this configuration the model predicts Lorenz-like dynamics. For parallel polarizations the model describes a markedly different dynamics which is studied in Sec. IV. These predictions for the two polarization configurations are consistent with previous experimental observations [5-10,28,29]. A closer fitting of theory and experiment requires the inclusion in the model of the Doppler broadening, the real degeneracy of levels (much larger than the one considered here), and probably other physical factors mentioned above and is therefore beyond the aim of this paper. Section V is devoted to conclusions and outlook.

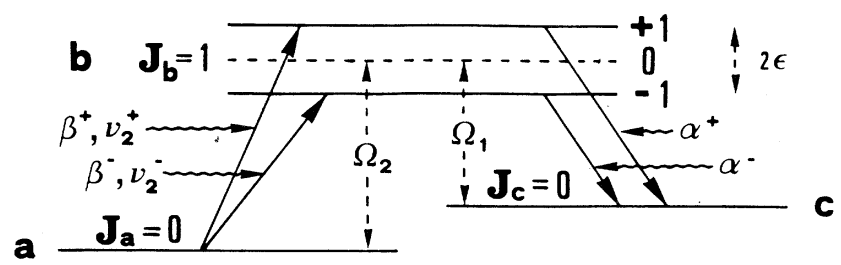

FIG. 1. Schematic representation of the level system and laser fields considered in this work. $2 \beta^{ \pm}$represent the pump Rabi frequencies and $2 \alpha^{ \pm}$the generated ones. $2 \varepsilon$ is the Zeeman splitting which is taken to be zero along this paper.

\section{HOMOGENEOUSLY BROADENED FOUR-LEVEL MODEL FOR AN OPML WITH LINEARLY POLARIZED FIELDS}

To take into account the vectorial character of the laser fields in an OPML one has to consider also the angular momentum quantum number of the radiatively coupled molecular states. Usually the total angular momentum quantum number $J$ of the three coupled levels is high (e.g., $J \simeq 7$ in Ref. [9]; $J \simeq 25$ in Ref. [27]) and therefore the total number $N \simeq 3(2 J+1)$ of magnetic sublevels involved is too large to be considered in a simple laser model. Instead, we study here a homogeneously broadened ring OPML that operates with the transition scheme $J_{a}=0 \rightarrow J_{b}=1 \rightarrow J_{c}=0$ shown in Fig. 1 [31]. It is the simplest level scheme that enables one to consider interaction with pump $\mathbf{E}_{2}(z, t)$ and generated $\mathbf{E}_{1}(z, t)$ fields composed by arbitrary superpositions of right and left circularly polarized components. Both fields are considered as uniform plane monochromatic waves propagating along the optical $(z)$ axis of the ring resonator

$$
\begin{aligned}
& E_{j}(z, t)=\sum_{\mu= \pm} \mathrm{e}^{\mu} E_{j}^{\mu}(z, t)+\text { c.c. } \quad(j=1,2), \\
& E_{1}^{\mu}(z, t)=\frac{1}{2} A_{1}^{\mu}(t) \exp \left\{i\left[k_{1} z-v_{1}^{\mu} t-\phi_{1}^{\mu}(t)\right]\right\}, \\
& E_{2}^{\mu}(z, t)=\frac{1}{2} A_{2}^{\mu} \exp \left\{i\left[k_{2} z-v_{2}^{\mu} t-\phi_{2}^{\mu}\right]\right\},
\end{aligned}
$$

where the unit vectors $\mathrm{e}^{ \pm}=\mp\left(\mathbf{e}_{x}+i \mathbf{e}_{y}\right) / \sqrt{2}$. The $z$ axis is also chosen to be the quantization axis, so that only four levels are involved [the sublevel $\left(J_{b}=1, m_{J}=0\right)$ does not couple to the fields], which we shall simply denote as $a,+,-$, and $c$. In the following we restrict ourselves to the case of $M$-degenerated levels $(\varepsilon=0)$ and fully resonant pump field $v_{2}^{+}=v_{2}^{-} \equiv \Omega_{2}$ (see Fig. 1). The amplitudes $A_{2}^{\mu}$ and phases $\phi_{2}^{\mu}$ of the pump beam are considered as timeindependent control parameters. For the reference frequencies of the generated fields we choose $v_{1}^{+}=v_{1}^{-} \equiv \omega_{c}$, $\omega_{c}$ being the closest empty cavity resonance frequency. The unknown amplitudes $A_{1}^{\mu}(t)$ and phases $\phi_{1}^{\mu}(t)$ of the generated beam are slowly varying functions of time. We assume that the pump beam (lasing beam) drives the $a-b$ $(b-c)$ transition only. Therefore the field-matter coupling is characterized by four real Rabi frequencies (see Fig. 1) defined as $\beta^{\mu}=d_{2} A_{2}^{\mu} / 2 \hbar$ and $\alpha^{\mu}=d_{1} A_{1}^{\mu} / 2 \hbar$ with $d_{j}$ $(j=1,2)$ being molecular transition electric dipole moments. It is worth noting that by taking $\beta^{-}=\alpha^{-}=0$ (or alternatively $\beta^{+}=\alpha^{+}=0$ ), one recovers the three-level model discussed in Refs. [16], [19], and [21], where attention was addressed to the influence of pump coherence on the laser dynamics.

As is typical in far-infrared-laser experiments we only consider in this paper pump and laser fields with fixed linear polarizations either parallel or crossed. Without loss of generality we take the generated field to be $e_{y}$ polarized, thus we have $\alpha^{+}(t)=\alpha^{-}(t) \equiv \alpha(t)$ and $\phi_{1}^{+}(t)=\phi_{1}^{-}(t) \equiv \phi(t)$. For the pump beam we have $\phi_{2}^{+}=\phi_{2}^{-} \equiv \phi_{2}$ and either $\beta^{+}=\beta^{-} \equiv \beta$ for the parallel case, or $\beta^{+}=-\beta^{-} \equiv \beta$ for the orthogonal case. Making use of these relations in Eqs. (2), (3), and (4) of Ref. [31], and assuming equal losses for both components of the generated 
fields $\left(\gamma^{+}=\gamma^{-} \equiv 2 \kappa\right)$, one easily obtains the relations

$$
\begin{aligned}
& \rho_{c+}(t)=\rho_{c-}(t) \equiv \rho_{c b}(t), \\
& \alpha\left[\left(\rho_{++}-\rho_{--}\right)-2 i \operatorname{Im}\left(\rho_{+-}\right)\right]=\left(\beta^{+}-\beta^{-}\right) \rho_{c a},
\end{aligned}
$$

which will be used later, and the following closed set of Maxwell-Schrödinger equations that govern the dynamics of the OPML with linearly polarized pump and laser fields:

$$
\begin{aligned}
\dot{\rho}_{a a}= & \gamma_{a}\left(\rho_{a a}^{0}-\rho_{a a}\right)+2 \beta^{+} y_{+a}+2 \beta^{-} y_{-a} \\
\dot{\rho}_{\mu \mu}= & \gamma_{\|}\left(\rho_{\mu \mu}^{0}-\rho_{\mu \mu}\right)+2 \alpha y_{c b}-2 \beta^{\mu} y_{\mu a} \\
\dot{\rho}_{c c}= & \gamma_{\|}\left(\rho_{c c}^{0}-\rho_{c c}\right)-4 \alpha y_{c b} \\
\dot{\rho}_{\mu a}= & -\gamma_{a b} \rho_{\mu a}+i\left(\beta^{-\mu} \rho_{-\mu \mu}^{*}+\beta^{\mu} N_{\mu a}\right)-i \alpha \rho_{c a} \\
\dot{\rho}_{c b}= & -\left[\gamma_{\perp}+i\left(\Delta_{1}^{c}+\dot{\phi}\right)\right] \rho_{c b}-i \alpha\left[\left(\rho_{++}+\rho_{--}\right) / 2-\rho_{c c}\right] \\
& \quad-i \alpha x_{+-}+i\left(\beta^{+}+\beta^{-}\right) \rho_{c a} / 2 \\
\dot{\rho}_{c a}= & -\left[\gamma_{a c}+i\left(\Delta_{1}^{c}+\dot{\phi}\right)\right] \rho_{c a}+i\left(\beta^{+}+\beta^{-}\right) \rho_{c b} \\
& \quad-i \alpha\left(\rho_{+a}+\rho_{-a}\right) \\
\dot{\rho}_{+-}= & -\Gamma \rho_{+-}-i\left(\beta^{+} \rho_{-a}^{*}-\beta^{-} \rho_{+a}\right)+2 \alpha y_{c b} \\
\dot{\alpha}= & -\kappa \alpha-g y_{c b}, \\
\dot{\phi}= & g x_{c b} / \alpha
\end{aligned}
$$

where $\mu=+,-; \rho_{j j}$ represents the population of level $j$; $N_{i j} \equiv\left(\rho_{i i}-\rho_{j j}\right)$ is a population difference; and $\rho_{i j}$ is the slowly varying complex amplitude of the coherence associated with the transition $i \rightarrow j \quad(i, j=a,+,-, c)$, $x_{i j} \equiv \operatorname{Re}\left(\rho_{i j}\right), y_{i j} \equiv \operatorname{Im}\left(\rho_{i j}\right)$. Dissipative processes are described by means of population relaxation rates $\gamma_{j}$ and coherence relaxation rates $\gamma_{i j}$. To allow a direct comparison with previous three-level results the values for the relaxation rates given in Ref. [21] will be adopted: $\gamma_{c+}=\gamma_{c-} \equiv \gamma_{\perp}=6.8 \times 10^{6} \quad \sec ^{-1} ; \quad \gamma_{+}=\gamma_{-}=\gamma_{c} \equiv \gamma_{\|}$ $=0.28 \gamma_{\perp} ; \gamma_{a}=\gamma_{a c}=0.95 \gamma_{\perp} ; \gamma_{a+}=\gamma_{a-} \equiv \gamma_{a b}=0.95 \gamma_{\perp}$. For $\gamma_{+-} \equiv \Gamma$ no experimental estimation of its value exists and we will explore a range of values $0.28 \gamma_{\perp} \leq \Gamma \leq 2.0$. For the populations of levels in the absence of fields we will assume [21,31] $\rho_{a a}^{0}: \rho_{++}^{0}: \rho_{--}^{0}: \rho_{c c}^{0}=0.9594: 0.0113: 0.0113: 0.0180 . \quad \Delta_{1}^{c}$ $\equiv \omega_{c}-\Omega_{1}$ is the cavity detuning and $g \equiv \Omega_{1} d_{1}^{2} N_{0} / 2 \varepsilon_{0} \hbar$ represents the unsaturated gain parameter, $N_{0}$ being the number of active molecules per unit volume. Note that Eq. (3.5) corresponds to the symmetrized expression $\dot{\rho}_{c b}=\left(\dot{\rho}_{c+}+\dot{\rho}_{c-}\right) / 2$, since $(2.1)$ is verified.

As indicated above, Eqs. (3) are valid for both parallel and crossed linear polarizations. Next we will elaborate on each one of these cases separately.

\section{ORTHOGONAL CASE}

\section{A. Model}

In this section we will consider the interaction between a linearly polarized pump beam ( $\mathbf{e}_{x}$ polarized) and an orthogonally polarized generated field (e $e_{y}$ polarized). For this case one has $\beta^{+}=-\beta^{-} \equiv \beta$. By introducing this con- dition into Eqs. (3.4) and making use of Eqs. (2) the following relations are obtained (see Appendix):

$$
\begin{aligned}
& \rho_{+a}(t)=\rho_{-a}^{*}(t) \equiv \rho_{b a}(t), \\
& \rho_{c a}(t)=0, \\
& x_{b a}(t)=0, \\
& y_{+-}(t)=0, \\
& \rho_{++}(t)=\rho_{--}(t) \equiv \rho_{b b}(t) .
\end{aligned}
$$

Making use of relations (4), Eqs. (3) can be written in the following form:

$$
\begin{aligned}
& \dot{\rho}_{a a}=\gamma_{a}\left(\rho_{a a}^{0}-\rho_{a a}\right)+4 \beta y_{b a}, \\
& \dot{\rho}_{b b}=\gamma_{\|}\left(\rho_{b b}^{0}-\rho_{b b}\right)+2 \alpha y_{c b}-2 \beta y_{b a}, \\
& \dot{N}_{b c}=\gamma_{\|}\left[\left(\rho_{b b}^{0}-\rho_{c c}^{0}\right)-N_{b c}\right]+6 \alpha y_{c b}-2 \beta y_{b a}, \\
& \dot{y}_{b a}=-\gamma_{a b} y_{b a}+\beta\left(N_{b a}-x_{+-}\right), \\
& \dot{\rho}_{c b}=-\left[\gamma_{\perp}+i\left(\Delta_{1}^{c}+\dot{\phi}\right)\right] \rho_{c b}-i \alpha\left(N_{b c}+x_{+-}\right), \\
& \dot{x}_{+-}=-\Gamma x_{+-}+2 \beta y_{b a}+2 \alpha y_{c b}, \\
& \dot{\alpha}=-\kappa \alpha-g y_{c b}, \\
& \dot{\phi}=g x_{c b} / \alpha,
\end{aligned}
$$

where $N_{b c} \equiv\left(\rho_{b b}-\rho_{c c}\right)$. Note that $N_{b c}$ involves only half the total population of the upper lasing levels $\left(\rho_{++}+\rho_{--}\right)$.

Relation (4.2) is of remarkable relevance since it means that in the orthogonal configuration there is no coherent coupling between the pump field and the laser field, which occurs in general via the two-photon coherence $\rho_{c a}$. Therefore, no Raman processes occur at all, nor pump-induced Rabi splitting of the laser gain. There remains only an incoherent coupling between pump and laser fields through the populations of levels + and and the coherence $x_{+-}$between them. This absence of Raman processes can be interpreted [32] as a destructive quantum interference between the two Raman channels $a \rightarrow+\rightarrow c$ and $a \rightarrow-\rightarrow c$. On the other hand, relation (4.3) implies that the refractive index at the pump frequency is not modified for any cavity detuning, at variance of what happens in the simpler three-level OPML model [21].

\section{B. Stationary solutions}

Equations (5) provide two different classes of stationary solutions. One of them corresponds to the nonlasing state with $\alpha=0$. A linear stability analysis (LSA) of this trivial solution shows that by increasing the pump intensity $\beta^{2}$ it destablizes through a pitchfork bifurcation occurring at [33]

$$
\beta^{2}=\beta_{1 \mathrm{st}}^{2} \equiv B / A \text {, }
$$

where

$$
A=2 g\left(\rho_{a a}^{0}-\rho_{b b}^{0}\right) \gamma_{a}\left(\Gamma-\gamma_{\|}\right)-2 \xi\left(2 \gamma_{\|} \Gamma+\gamma_{\|} \gamma_{a}+\gamma_{a} \Gamma\right),
$$


$B=\gamma_{\|} \gamma_{a} \gamma_{a b} \Gamma \xi$

$\xi=g\left(\rho_{c c}^{0}-\rho_{b b}^{0}\right)+\kappa\left[1+\left[\frac{\Delta_{1}^{c}}{\kappa+1}\right]^{2}\right]$.

Since $\beta$ is a real quantity Eq. (6) can only be fulfilled if the three following conditions are met:

$$
\begin{aligned}
& g \geq g_{\mathrm{thr}} \equiv \kappa\left(\rho_{a a}^{0}-\rho_{c c}^{0}\right)\left(2 \gamma_{\|} \Gamma+\gamma_{\|} \gamma_{a}+\gamma_{a} \Gamma\right) \\
& \times\left[1+\left[\frac{\Delta_{1}^{c}}{\kappa+1}\right]^{2}\right] \frac{\gamma_{a}-\gamma_{a}^{\mathrm{thr}}}{\Gamma-\Gamma_{\mathrm{thr}}}, \\
& \Gamma \geq \Gamma_{\mathrm{thr}} \equiv \frac{1-3 \rho_{b b}^{0}}{\rho_{a a}^{0}-\rho_{c c}^{0}} \frac{\gamma_{a} \gamma_{\|}}{\gamma_{a}-\gamma_{a}^{\mathrm{thr}}}, \\
& \gamma_{a} \geq \gamma_{a}^{\mathrm{thr}} \equiv 2 \gamma_{\|}\left(\rho_{c c}^{0}-\rho_{b b}^{0}\right) /\left(\rho_{a a}^{0}-\rho_{c c}^{0}\right) .
\end{aligned}
$$

In our case $\gamma_{a}^{\mathrm{thr}}=0.004$ and $\Gamma_{\mathrm{thr}}=0.285$. The variation of the pump intensity threshold for cw laser emission $\beta_{1 \mathrm{st}}^{2}$, with the relaxation rate $\Gamma$ as a function of the cavity detuning $\Delta_{1}^{c}$, is shown in Fig. 2, where one can observe the rapid increase of $\beta_{1 \mathrm{st}}^{2}$ as $\Gamma$ approaches $\Gamma_{\mathrm{thr}}$.

The stationary lasing solution that bifurcates from the nonlasing state when $\beta^{2}>\beta_{1 \text { st }}^{2}$ is given by

$$
\alpha^{2}=\frac{\gamma_{\|}}{2 \kappa} \frac{A \beta^{2}-B}{C \beta^{2}+H}
$$

with

$$
\begin{aligned}
& C=4 \gamma_{\|}\left(\gamma_{\|}+3 \Gamma\right)+4 \gamma_{a}\left(3 \gamma_{\|}+\Gamma\right), \\
& H=\gamma_{\|} \gamma_{a} \gamma_{a b}\left(\gamma_{\|}+3 \Gamma\right),
\end{aligned}
$$

and $A$ and $B$ given by Eqs. (7).

The pulling effect corresponding to this lasing solution is given by

$$
\dot{\phi}=-\kappa \Delta_{1}^{c} /(\kappa+1),
$$

which is identical to that of a two-level homogeneously broadened ring laser (Lorenz-Haken laser) [13,34] as a

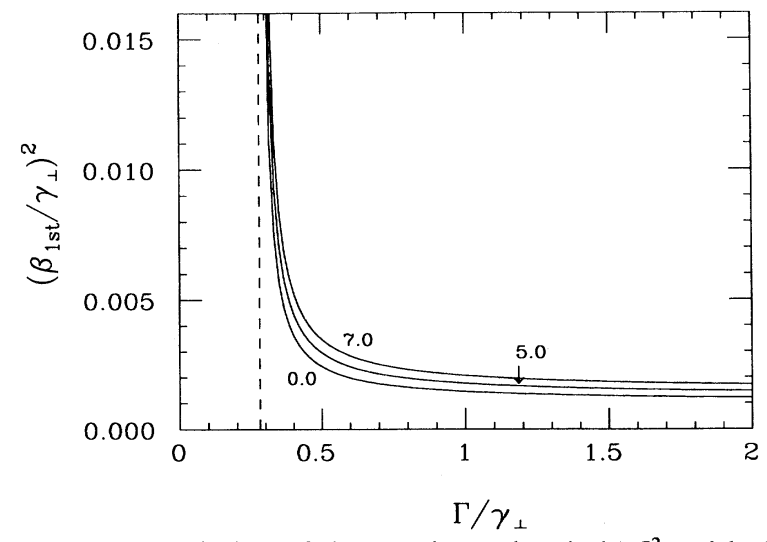

FIG. 2. Variation of the first laser threshold $\beta_{1 \text { st }}^{2}$ with the relaxation rate $\Gamma$ as a function of the cavity detuning $\Delta_{1}^{c}$ for $g / \gamma_{\perp}^{2}=3642.5, \kappa / \gamma_{\perp}=2$, and crossed polarizations for pump and generated fields. Different curves from top to bottom correspond to $\Delta_{1}^{c} / \gamma_{\perp}=7,5$, and 0 . Other parameters are given in the text. consequence of the lack of coherent pumping effects in the orthogonal configuration. For the sake of brevity the stationary value of the rest of variables is not given here. [Only the stationary value of $x_{+-}$is given below in Eq. (15).]

Figure 3 shows the variation of the steady laser intensity $\alpha^{2}$ as a function of the pump intensity $\beta^{2}$, given by Eq. (9), for the case $\Delta_{1}^{c}=0$ and several values of $\Gamma$ in Fig. 3(a), and for $\Gamma=0.5$ and different values of the detuning $\Delta_{1}^{c}$ in Fig. 3(b). In both figures it is clearly apparent the nonlinear variation of the laser intensity with $\beta^{2}$ at variance of what happens for an ordinary incoherently pumped two-level laser. Although the optical pumping does not introduce coherent effects in this orthogonal configuration [see Eq. (4.2) and subsequent discussion], there remains a nonlinear dependence due to pump field absorption saturation. On the other hand, and at difference with the behavior of the usual three-level OPML, now there is not an upper limit for the pump in-
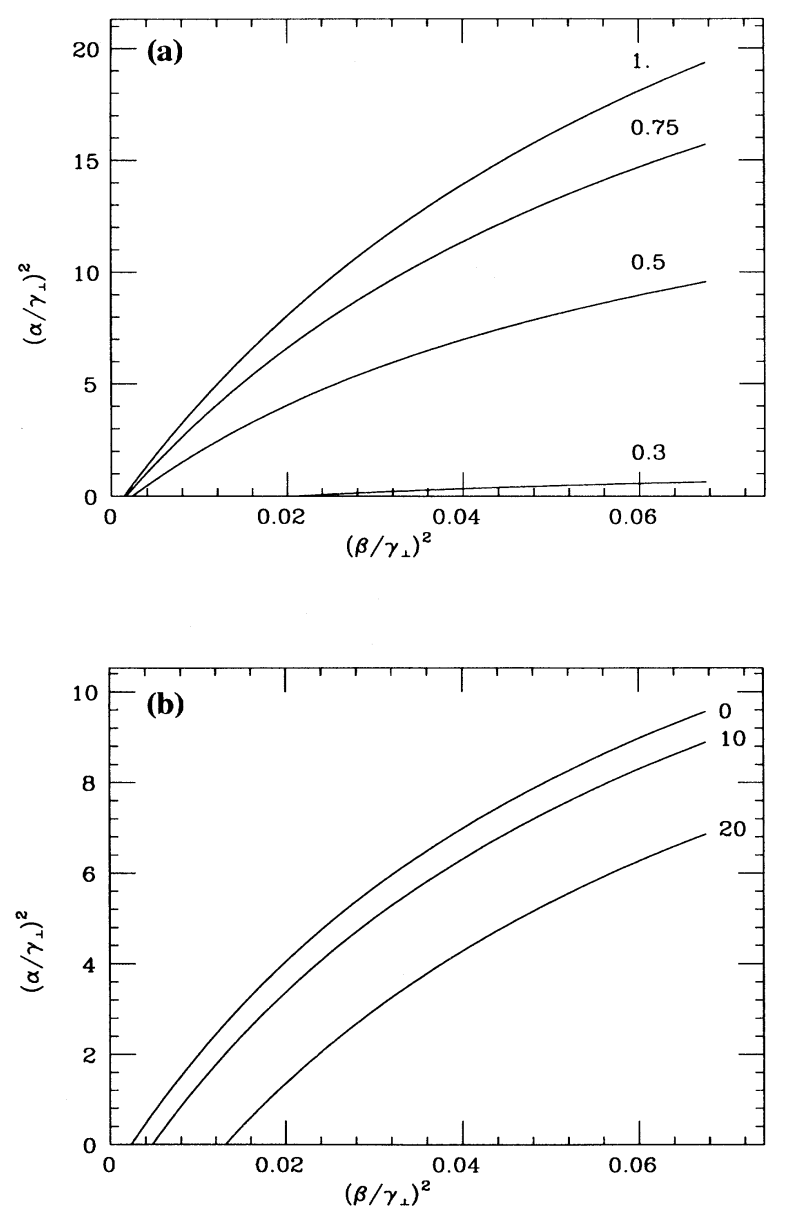

FIG. 3. Variation of the steady laser intensity $\alpha^{2}$ as a function of the pump intensity $\beta^{2}$ for crossed polarizations. For (a) $\Delta_{1}^{c}=0$ and different curves from top to bottom correspond to $\Gamma / \gamma_{\perp}=1,0.75,0.5$, and 0.3 . For (b) $\Gamma / \gamma_{\perp}=0.5$ and curves from top to bottom correspond to $\Delta_{1}^{c} / \gamma_{\perp}=0,10$, and 20. (Other parameters are as in Fig. 2.) 

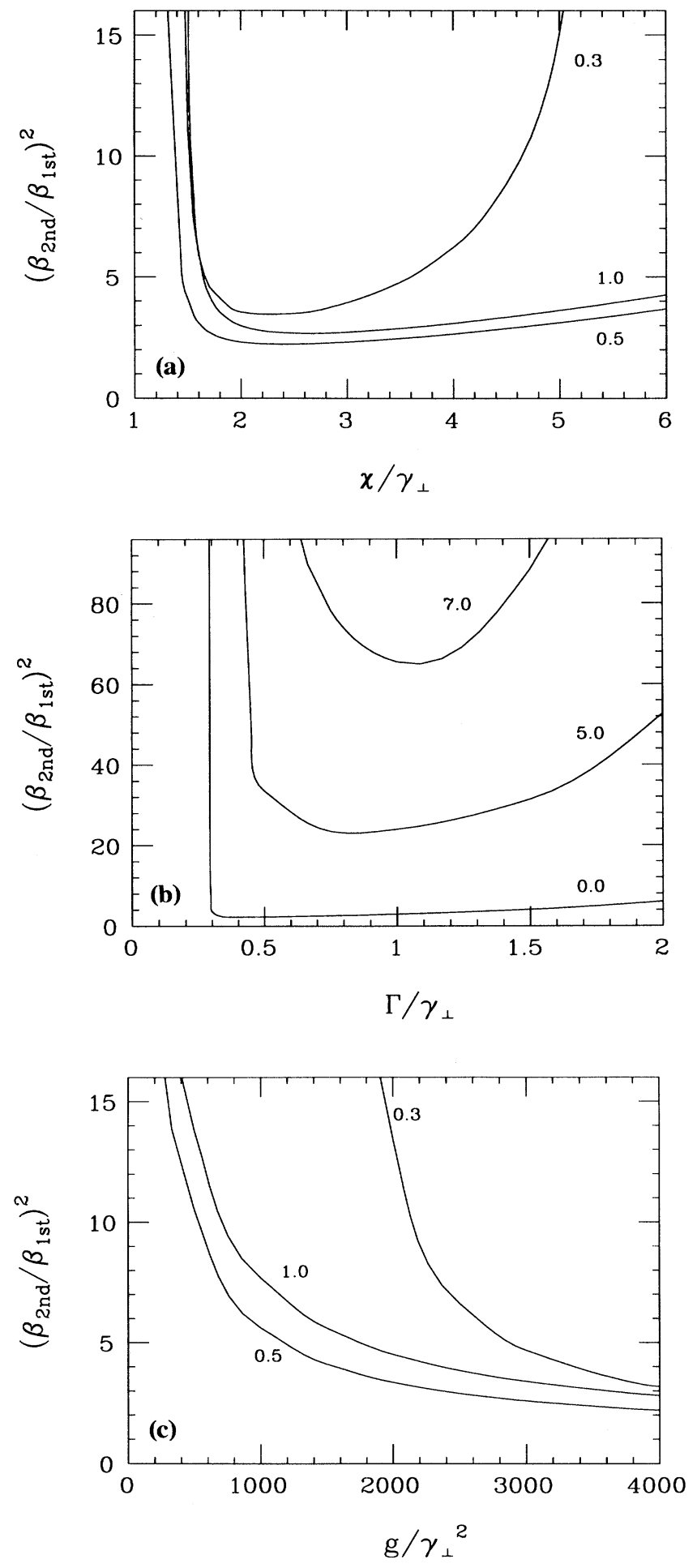

FIG. 4. Ratio between threshold pump intensities for softmode laser instabilities $\beta_{2 \text { nd }}$ and for cw laser emission $\beta_{1 \mathrm{st}}$ in the crossed-polarization configuration, as a function of (a) cavity losses $\kappa$ for $g / \gamma_{\perp}^{2}=3642.5$ and $\Delta_{1}^{c}=0$ and curves from top to bottom corresponding to $\Gamma / \gamma_{\perp}=0.3,1$, and 0.5 ; (b) relaxation rate $\Gamma$ for $\kappa / \gamma_{\perp}=2, g / \gamma_{\perp}^{2}=3642.5$, and curves from top to bottom corresponding to $\Delta_{1}^{c} / \gamma_{\perp}=7,5$, and 0 ; (c) gain parameter $g$ for $\kappa / \gamma_{\perp}=2, \Delta_{1}^{c}=0$, and several values of $\Gamma / \gamma_{\perp}$ as in (a). tensity after which the laser turns off [19,21]. This is a consequence of the absence of Rabi splitting of the laser gain.

A LSA of the stationary solution (9) allows us to determine the threshold for soft-mode laser instabilities (instability threshold or second laser threshold $\left.\beta_{2 n d}^{2}\right)$. The ratio $\left(\beta_{2 \text { nd }} / \beta_{1 \text { st }}\right)^{2}$ is shown in Fig. 4 as a function of several laser parameters. Three main features can be observed. (i) The ratio $\left(\beta_{2 \mathrm{nd}} / \beta_{1 \mathrm{st}}\right)^{2}$ tends to infinity for $\Gamma=\Gamma_{\text {thr }}[\mathrm{Fig}$. 4(b)] and for $g=g_{\text {thr }}$ [Fig. 4(c)]. (ii) Instabilities appear only [Fig. $4(\mathrm{a})$ ] for $\kappa / \gamma_{\perp} \geq 1.35>\kappa_{\mathrm{BC}}=1+\gamma_{\|} / \gamma_{\perp}=1.28$, i.e., the "bad cavity" condition must be fulfilled. (iii) The curves in Figs. 4(a) and 4(c) exhibit a nonmonotonic dependence on $\Gamma$.

\section{Dynamics}

To further characterize the unstable behavior of our system we have numerically integrated Eqs. (5) using a seventh-eight-order Runge-Kutta routine. The phase diagrams of Fig. 5 show the different emission regimes of the laser intensity $\alpha^{2}(t)$, obtained by hard-mode excitation, in the parameter plane $\left(\beta, \Delta_{1}^{c}\right)$. The dashed line in Fig. 5(a) corresponds to the second laser threshold $\beta_{2 \text { nd }}$ obtained with the LSA. It is apparent the existence of a certain domain of dynamic behavior before the system has reached the second threshold. This is possible because in this region (delimited by the continuous line and the dashed line) there is a coexistence of two attractors (generalized bistability). One of them is the steady-state
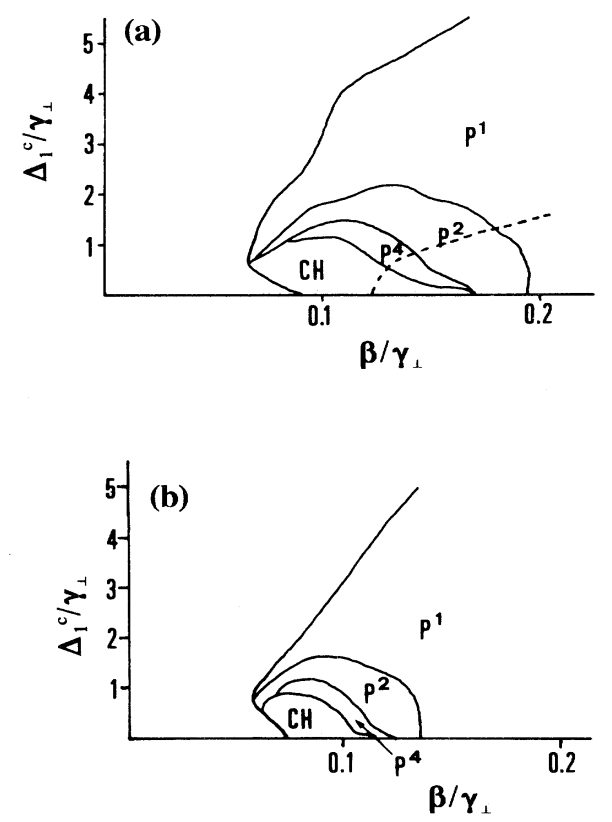

FIG. 5. Phase diagrams of the coherently pumped laser intensity $\alpha^{2}$ in the parameter plane $\left(\beta, \Delta_{1}^{c}\right)$, for crossed linear polarizations of pump and generated fields. Continuous lines (dashed lines) correspond to results obtained with hard-mode (soft-mode) excitation. For (a) $\Gamma / \gamma_{\perp}=0.5$, (b) 1 (other parameters as in Fig. 2). No soft-mode laser instability threshold is found for (b). 
fixed point and the other corresponds to the dynamical solutions. For the range of parameters explored in Fig. 5(b) the LSA indicates that the fixed point is always stable. Therefore, the generalized bistability occurs now for all the dynamic regimes shown in Fig. 5(b). As can be seen in this Fig. 5 there are periodic and chaotic regimes, with transitions to chaos occurring for increasing pump field strength $\beta$ and for increasing cavity detuning $\Delta_{1}^{c}$
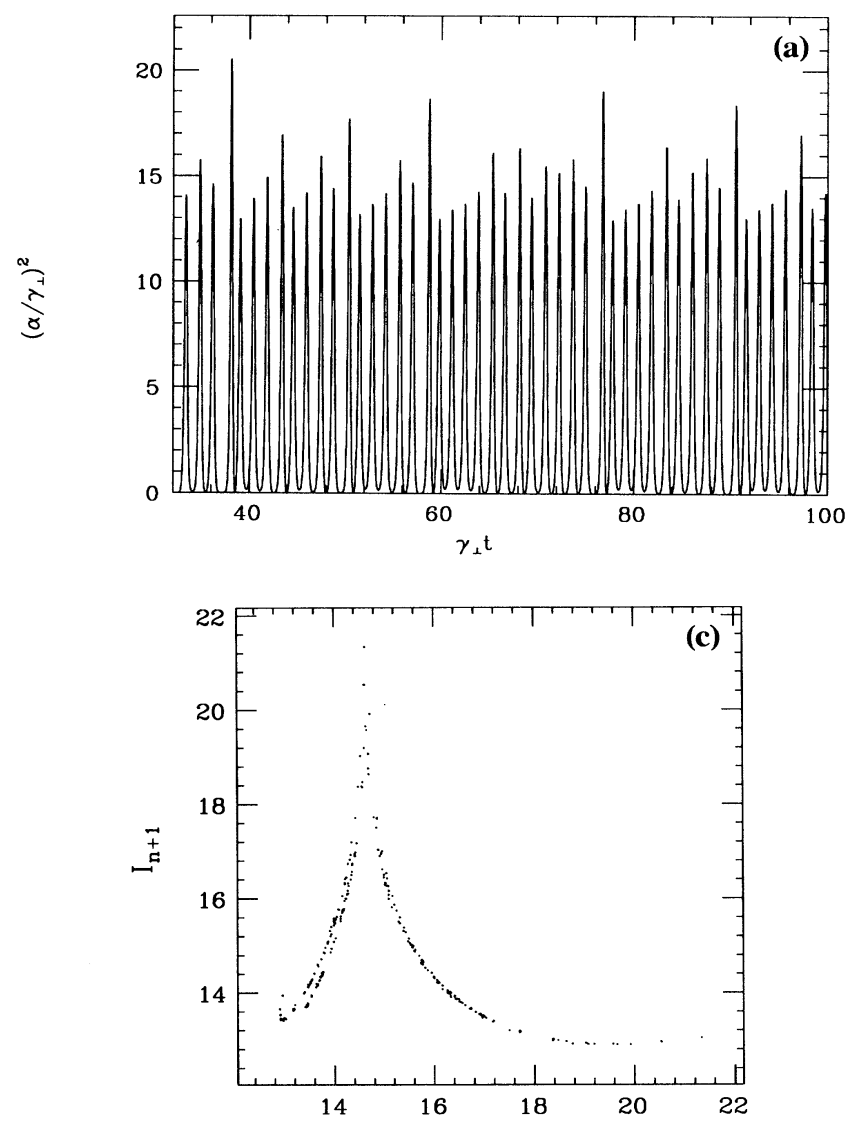

$I_{n}$

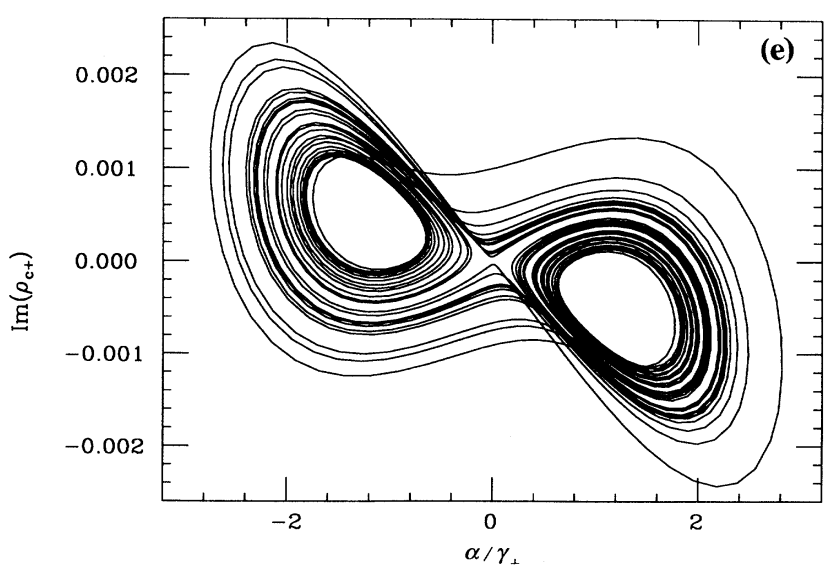

through an inverse sequence of period-doubling bifurcations as in the Lorenz-Haken model [13].

Representative chaotic time series of the laser intensity are shown in Fig. 6 for three decreasing values of the coherence relaxation rate $\Gamma=2,0.5$, and 0.295 , approaching $\Gamma_{\text {thr }}$, together with a phase portrait on the fieldpolarization plane and the corresponding intensity map. As seen the attractor appears with the butterfly shape
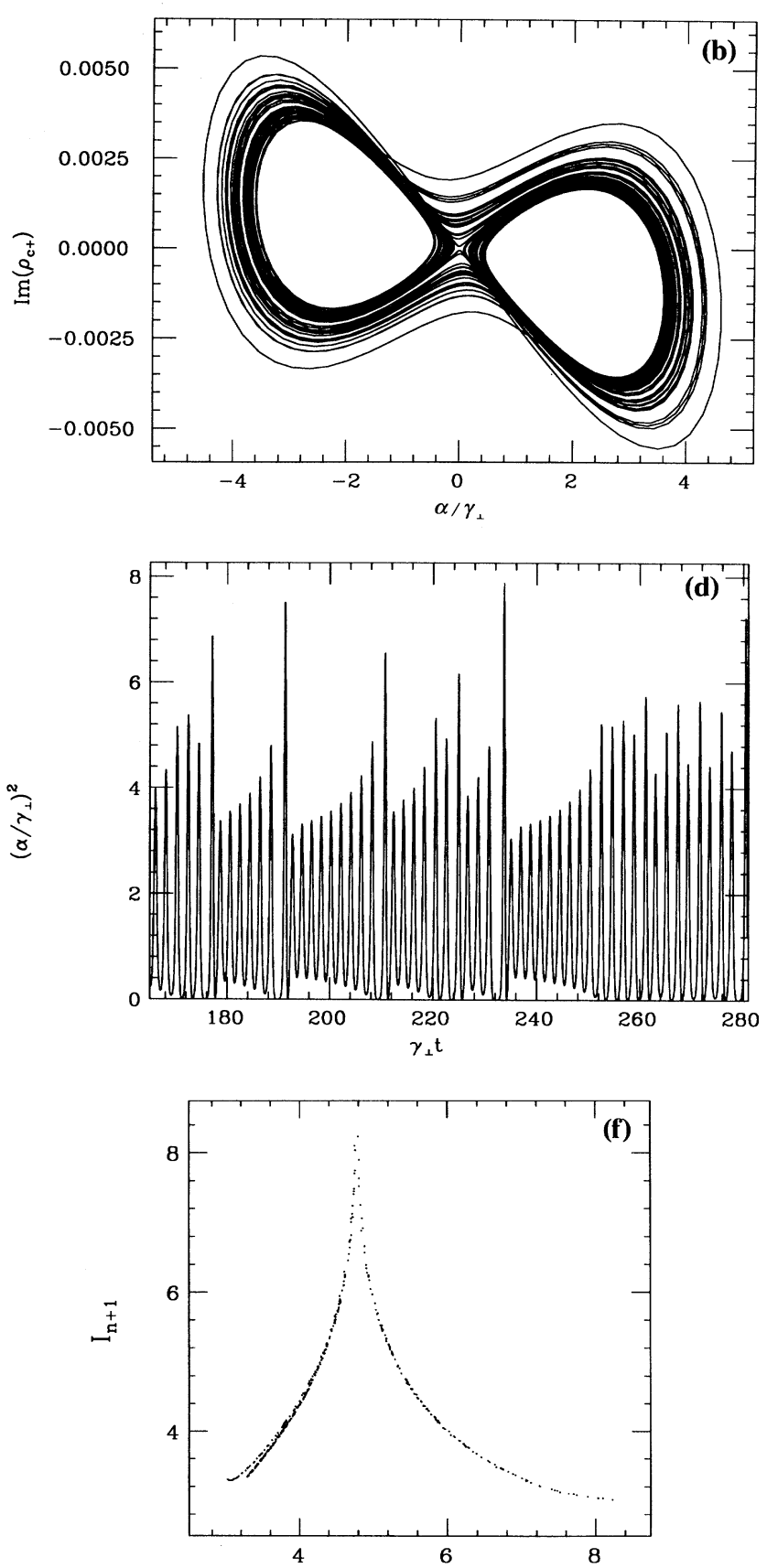

$I_{n}$

FIG. 6. Intensity pulsings $\alpha^{2}(t)$ in the chaotic attractor of Fig. 5, together with the respective two-dimensional $\left(\alpha, \operatorname{Im} \rho_{c+}\right)$ projections and the intensity return maps $I_{n+1}\left(I_{n}\right)$ (peak intensity of laser emission pulse $n+1$ vs peak intensity of pulse $n$ ). The values of $\Gamma / \gamma_{\perp}$ and $\beta / \gamma_{\perp}$ are 2 and 0.08 , respectively, for (a), (b), and (c); 0.5 and 0.9 for (d), (e), and (f); 0.295 and 10 for (g), (h), and (i). The pumps are close to the respective instability thresholds. 

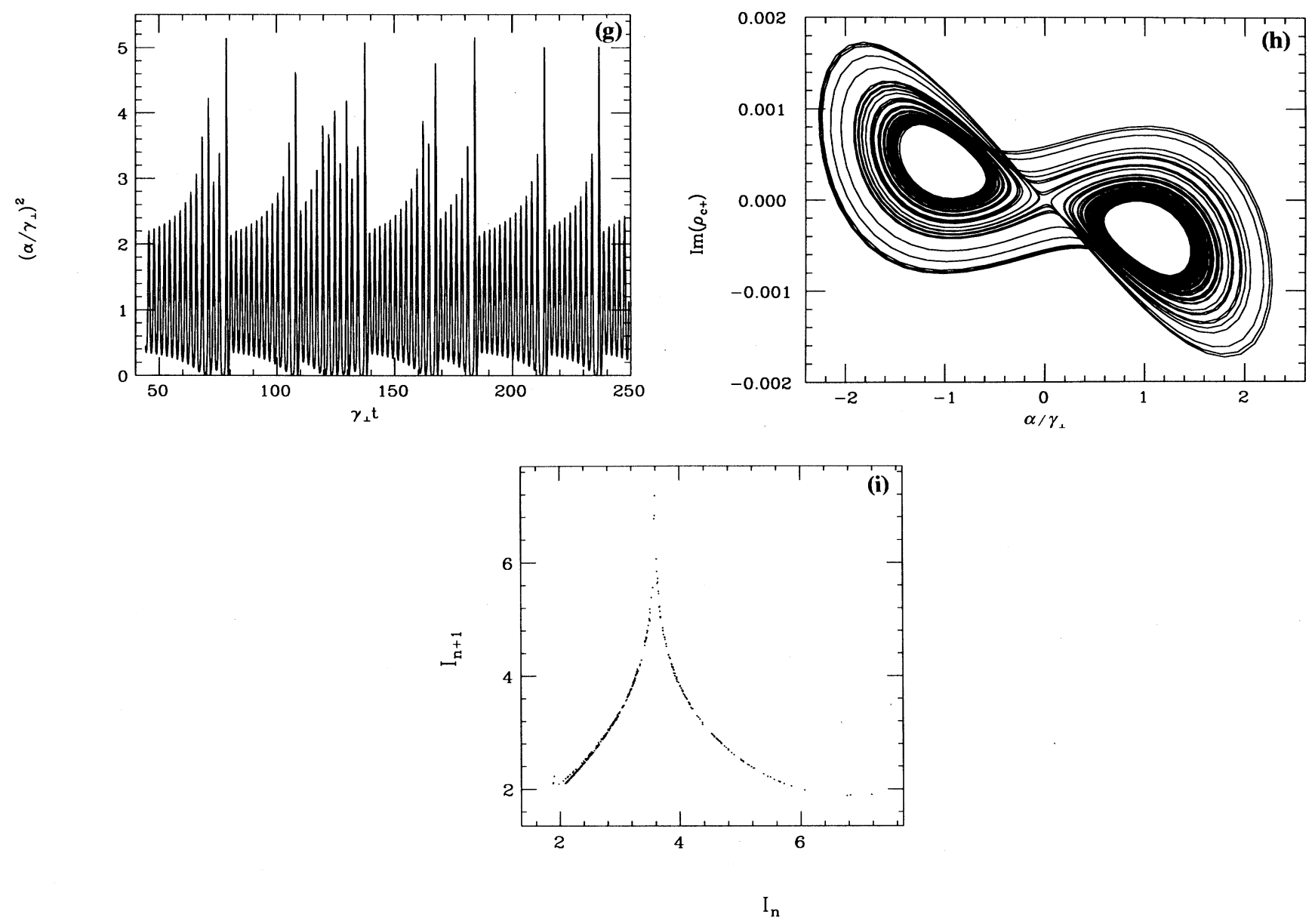

FIG. 6. (Continued).

characteristic of the Lorenz-Haken model, and $\alpha^{2}(t)$ shows the typical "spiralings" of this model [12]. This type of motion produces the "cusp"-shaped laser intensity map shown in Figs. 6(c), 6(f), and 6(i) [12,8].

It is worth pointing out that all these features found in our model are well-known characteristics of the LorenzHaken model [12]. Note also that the similarity between both models increases as $\Gamma$ gets closer to $\Gamma_{\text {thr }}$. For instance, for $\Gamma=2$ the "cusp" map of Fig. 6(c) presents a well-defined double "cusp" which does not appear in the Lorenz model for pump parameters close to the instability threshold. As $\Gamma$ decreases [Figs. 6(f) and 6(i)] this double "cusp" tends to disappear, approaching the Lorenz behavior. It is interesting to comment that in some intensity maps obtained from the experimental recordings $[8,9]$ a double "cusp" also appears. But it must be stressed that the origin of this second "cusp" is not the same as in our case. In the experiments, the second "cusp" was identified with the presence of an extra intermediate-height pulse at the beginning of each spiral [9]. On the contrary, in our case the first pulse at the beginning of each spiral seems to be too short as compared to what one should expect from the Lorenz model. The origin of the similarities and differences between our model and the Lorenz-Haken model is further discussed in the next subsection.

\section{Discussion}

Although neither the stationary solution given in Eq. (9) nor the LSA [11] behave as in a Lorenz-Haken laser (see Figs. 3 and 4, in particular, the stationary solution is nonlinear with respect to the pump parameter $\beta^{2}$ ), in Sec. III C it has been pointed out that the orthogonalpolarization OPML model and the Lorenz-Haken model $[12,13,35]$ exhibit qualitatively similar dynamical features. In order to further clarify this point let us compare in detail the equations of the two models.

To this purpose we introduce the following change of variables:

$$
\begin{aligned}
& E(t) \equiv \alpha(t) \exp [-i \phi(t)], \\
& P(t) \equiv i \rho_{c b}^{*}(t) \exp [-i \phi(t)], \\
& D(t) \equiv N_{b c}(t)+x_{+-}(t) .
\end{aligned}
$$

Then Eqs. (5) may be written as

$$
\begin{aligned}
& \dot{E}=-\kappa E-g P, \\
& \dot{P}=-\left(\gamma_{\perp}-i \Delta_{1}^{c}\right) P-E D, \\
& \dot{D}=\gamma_{\|}\left(D_{0}-D\right)+4\left(E P^{*}+P E^{*}\right)-\left(\Gamma-\gamma_{\|}\right) x,
\end{aligned}
$$


and

$$
\begin{aligned}
& \dot{x}=-\Gamma x+2 \beta y+\left(E P^{*}+P E^{*}\right), \\
& \dot{y}=-\gamma_{a b} y+\beta\left(\rho_{b b}-\rho_{a a}-x\right), \\
& \dot{\rho}_{a a}=\gamma_{a}\left(\rho_{a a}^{0}-\rho_{a a}\right)+4 \beta y, \\
& \dot{\rho}_{b b}=\gamma_{\|}\left(\rho_{b b}^{0}-\rho_{b b}\right)+\left(E P^{*}+P E^{*}\right)-2 \beta y,
\end{aligned}
$$

where $D_{0} \equiv\left(\rho_{b b}^{0}-\rho_{c c}^{0}\right)$, and use has been made of the simplified notations $x(t) \equiv x_{+-}(t), y(t) \equiv y_{b a}(t)$.

Equations (13.1)-(13.3) would be isomorphic to those of the complex Lorenz-Haken laser model [11] if the last term in the right-hand side of Eq. (13.3), i.e., $\left[-\left(\Gamma-\gamma_{\|}\right) x\right]$, were null. This is the only term that couples Eqs. (13.1)-(13.3) and Eqs. (13.4)-(13.7). Since the coherence decay rate $\Gamma$ may be written as $\Gamma=\left(\gamma_{++}+\gamma_{--}\right) / 2+\gamma_{\mathrm{ph}}=\gamma_{\|}+\gamma_{\mathrm{ph}}$, where the first contribution $\gamma_{\|}$is related to the decay of molecular states and $\gamma_{\mathrm{ph}}$ accounts for phase interrupting collisions, it follows that when $\Gamma=\gamma_{\|}$(i.e., the lowest value $\Gamma$ can take), the two-level variables $(E, P, D)$ become unaffected by the evolution of the rest of variables $\left(x, y, \rho_{a a}, \rho_{b b}\right)$, although the former influence the latter as can be seen in Eqs. (13). Moreover, since the optical pumping acts only directly on the variables $\left(x, y, \rho_{a a}, \rho_{b b}\right)$, the effective variables $(E, P, D)$ are blind to this pump, and are only determined by the initial population inversion $D_{0}$. Since $D_{0}$ is negative in the absence of an external incoherent pumping mechanism, the laser will not lase independently of the values of the pump strength $\beta^{2}$ and the gain parameter $g$. This result is in agreement with condition (8.2), which gives the minimum value of $\Gamma$ that permits lasing operation, since in the particular case we are discussing $\left(\Gamma=\gamma_{\|}\right), \Gamma$ is below $\Gamma_{\text {thr }}$.

Nevertheless, if one looks at the stationary population inversion corresponding to the lasing transition

$$
N_{b c}=\left(\rho_{b b}^{0}-\rho_{c c}^{0}\right)+\frac{2 \gamma_{a}\left(\rho_{a a}^{0}-\rho_{b b}^{0}\right) \beta^{2}}{\gamma_{\|} \gamma_{a} \gamma_{a b}+4\left(\gamma_{\|}+\gamma_{a}\right) \beta^{2}},
$$

which is plotted in Fig. 7 as a function of $\beta^{2}$ for $\Gamma=\gamma_{\|}$,

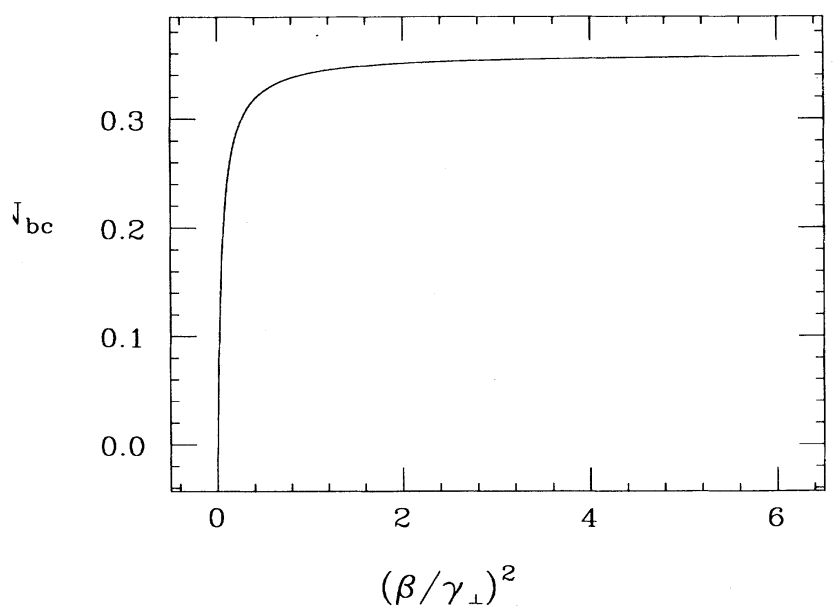

FIG. 7. Population inversion $N_{b C}$ as a function of pump intensity $\beta^{2}$ for $\Gamma=\gamma_{\|}$. Other parameters as in Fig. 4 .

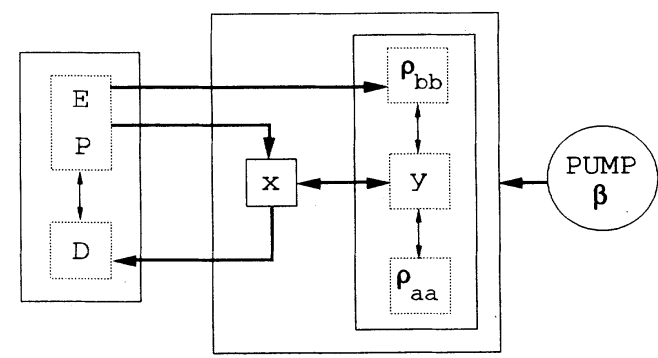

FIG. 8. Flow diagram showing schematically the coupling [see Eqs. (13)] between two-level laser variables $(E, P, D)$, the rest of variables $\left(x, \rho_{b b}, y, \rho_{a a}\right)$ and the pump field $\beta$.

one observes that there is a net population inversion. This inversion would give rise to emission for an appropriate value of the gain parameter $g$ in a normal twolevel laser, but in our case the laser remains off for any value of this parameter. This phenomenon of population inversion without lasing can be clearly interpreted in the context of the dressed-atom formalism $[32,36]$. Inversion between bare states does not guarantee inversion between states dressed by the pump beam. In Ref. [32] it is shown that population inversion between the dressed states coupled to the generated field is only possible if $\Gamma>\Gamma_{\mathrm{thr}}$. For $\Gamma<\Gamma_{\text {thr }}$ the population is trapped in dressed states not coupled to the laser field [32].

Up to now, we have shown that our crossedpolarization OPML model exactly reduces to the Lorenz-Haken model in the particular case in which $\Gamma$ takes its minimum value $\left(\Gamma=\gamma_{\|}\right)$and that in this situation our laser will not emit if the optical pumping of the adjacent transitions $a \rightarrow b$ is the only external pumping mechanism.

For $\Gamma>\Gamma_{\text {thr }}$, however, our model does not reduce to the Lorenz-Haken model, but rather to a modified one with the extra term $\left[-\left(\Gamma-\gamma_{\|}\right) x\right]$ in Eq. (13.3), which connects the dynamics of all the variables of the system. Nevertheless, it clearly appears in Fig. 8 that the role of the variable $x$ is basically that of an incoherent pumping mechanism on the lasing transition. This pumping, however, is coupled with the dynamics of the whole system. Figure 9 shows a typical time evolution of $x$ in the chaotic domain. It consists of a nonzero dc contribution $x_{\mathrm{dc}}$ and a superimposed modulation. For Fig. 9 $x_{\mathrm{dc}}=-0.0307$, which is close to its (unstable) stationary value $\bar{x}=-0.0318$ given by Eq. (15) below. The variation of $x$ around $x_{\mathrm{dc}}$ is less than $6 \%$. When one considers periodic behaviors, the value $x_{\mathrm{dc}}$ is closer to $\bar{x}$ than in the chaotic regime.

All these general features of the variable $x$ permit a first approximate treatment which reduces the complete model of Eqs. (13) to an effective Lorenz-Haken model. This can be done by substituting the actual instantaneous value of $x$ by its stationary value $\bar{x}$, which is given by

$$
\begin{gathered}
\bar{x}=-\frac{1}{\Gamma-\gamma_{\|}}\left\{\gamma_{\|}\left(\rho_{c c}^{0}-\rho_{b b}^{0}\right)+\frac{\kappa \gamma_{\|}}{g}\left[1+\left[\frac{\Delta_{1}^{c}}{\kappa+1}\right]^{2}\right]\right. \\
\left.+\frac{4 \gamma_{\|}}{g} \frac{A \beta^{2}-B}{C \beta^{2}+H}\right\},
\end{gathered}
$$




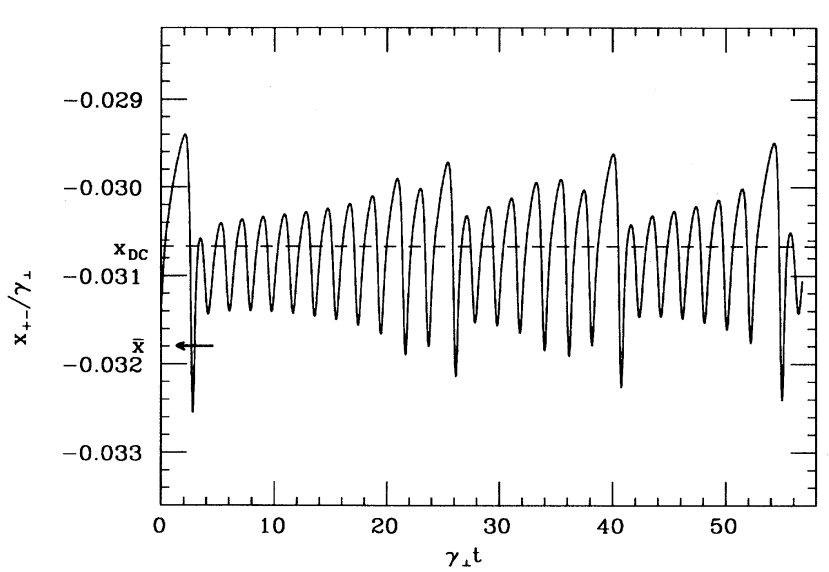

FIG. 9. Time evolution of the variable $x$ in the chaotic domain for $\Gamma / \gamma_{\perp}=0.5$ and $\beta / \gamma_{\perp}=0.09 . x_{\mathrm{dc}}$ denotes the average value of this variable, and $\bar{x}$ the (unstable) stationary value which is marked by an arrow.

where $A, B, C$, and $H$ are given as in Eqs. (7) and (10).

With this approximation the model reduces to Eqs. (13.1) and (13.2), and Eq. (13.3), which may be written as

$$
\dot{D}=\gamma_{\|}\left(D_{0}^{\text {eff }}-D\right)+4\left(E P^{*}+P E^{*}\right),
$$

with

$$
D_{0}^{\text {eff }} \equiv \frac{\kappa}{g}\left\{1+\left[\frac{\Delta_{1}^{c}}{\kappa+1}\right]^{2}+\frac{4}{\kappa} \frac{A \beta^{2}-B}{C \beta^{2}+H}\right\} .
$$

$D_{0}^{\text {eff }}$ is the effective incoherent pump parameter of the equivalent Lorenz-Haken model, which is nonlinear with respect to the optical pump intensity $\beta^{2}$, as can be seen in Fig. 10, where the normalized pump parameter $r \equiv D_{0}^{\text {eff }} g / \kappa$ (as defined for the Lorenz model) is plotted for different cavity relaxation rates $\kappa$. Note that $r$ saturates for $\beta^{2} \geq 1$ due to pump absorption saturation.

In order to test the degree of accuracy of this approxi-

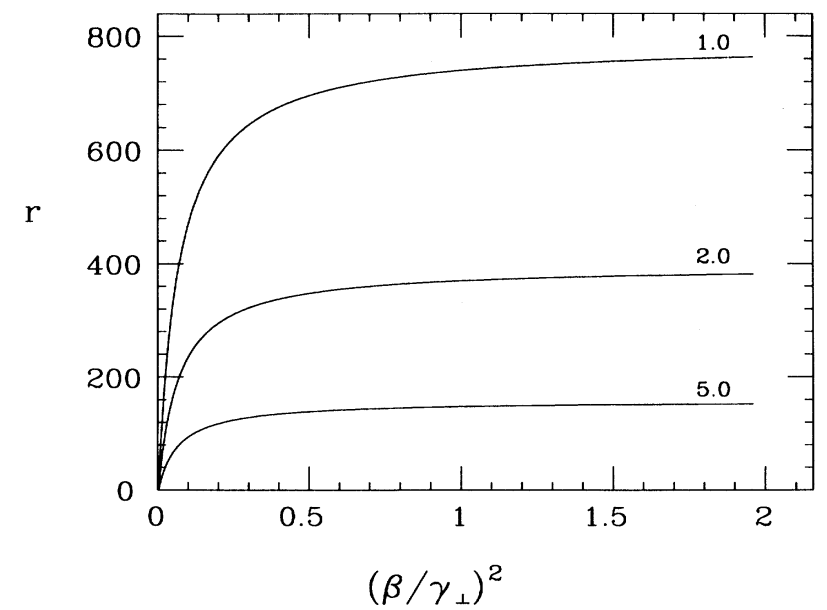

FIG. 10. Normalized pump parameter $r$ vs pump intensity $\beta^{2}$ for $\Delta_{1}^{c} / \gamma_{\perp}=0, g / \gamma_{\perp}^{2}=3642.5, \Gamma / \gamma_{\perp}=0.5$, and different values of $\kappa / \gamma_{\perp}=1,2$, and 5 . mation we compare now the LSA of the stationary lasing solutions of both the truncated system and the complete system, which was shown in Fig. 4. The LSA of the stationary lasing solution of the complex Lorenz-Haken model was performed by Zeghlache [37(a)], Mandel and Zeghlache [37(b)], and Bakasov and Abraham [37(c)]. By using the results of these works we obtain the equation that determines the value of the critical intensity $I_{c}=|E|^{2}=\alpha^{2}$ at which the threshold for instabilities is reached:

$$
c_{2} I_{c}^{2}+c_{1} I_{c}+c_{0}=0
$$

where the coefficients $c_{0}, c_{1}$, and $c_{2}$ are given in Refs. [37(a)] and [37(c)].

Substitution of Eq. (9) into Eq. (18) enables one to obtain the pump strength $\beta^{2}=\beta_{2 \text { nd }}^{2}$ at the threshold for instabilities. The results of this analysis are summarized in Fig. 11. A comparison of Figs. 4 and 11 shows that the approximate treatment reproduces fairly well the dependence of the ratio between threshold pump intensities on the cavity losses [Figs. 4(a) and 11(a)] and on the gain parameter $g$ [Figs. 4(c) and 11(c)] for $\Gamma=0.3$. For increasing $\Gamma$ one observes a monotonic variation of the curves in Figs. 11(a) and 11(c) at variance with what happens in Figs. 4(a) and 4(c) although the corresponding curves have qualitatively similar shapes. The largest discrepancies between the exact and the approximated results appear when one compares Figs. 4(b) and 11(b). Note the different vertical scales and also that corresponding curves have different shapes in both figures. This confirms that the truncation is a good approximation for values of $\Gamma$ close to $\Gamma_{\text {thr }}$, in agreement with the numerical results of Sec. III C.

A better approximate treatment should also include the modulation of the variable $x$ and not only its stationary value. This can be done along the lines described in Ref. [25] by introducing different effective values $\gamma_{\|}^{\text {eff }}$ and $D_{0}^{\text {eff }}$. We have tried also this additional approximate treatment and studied the corresponding LSA. In spite of a greatly increased numerical complexity the agreement with the exact treatment has not improved significantly.

\section{PARALlel CASE}

\section{A. Model}

In this section we will consider the interaction between pump and generated fields with parallel linear polarizations. For this case one has $\beta^{+}=\beta^{-} \equiv \beta$, which substituted into Eq. (2.2) leads to

$$
\begin{aligned}
& \rho_{++}(t)=\rho_{--}(t) \equiv \rho_{b b}(t), \\
& y_{+-}(t)=0 .
\end{aligned}
$$

On the other hand making use of Eqs. (2) and (19) and of the equations of evolution for $D_{+-}(t)$ $=\rho_{++}(t)-\rho_{--}(t)$ and $y_{+-}(t)$, we obtain

$$
\rho_{a+}(t)=\rho_{a-}(t) \equiv \rho_{a b}(t) \text {. }
$$

Finally, substitution of Eqs. (19) and (20) into Eqs. (3) 
allows us to write the equations which govern the dynamics of our system in the parallel configuration as

$$
\begin{aligned}
& \dot{\rho}_{a a}=\gamma_{a}\left(\rho_{a a}^{0}-\rho_{a a}\right)+4 \beta y_{b a}, \\
& \dot{\rho}_{b b}=\gamma_{\|}\left(\rho_{b b}^{0}-\rho_{b b}\right)+2 \alpha y_{c b}-2 \beta y_{b a},
\end{aligned}
$$
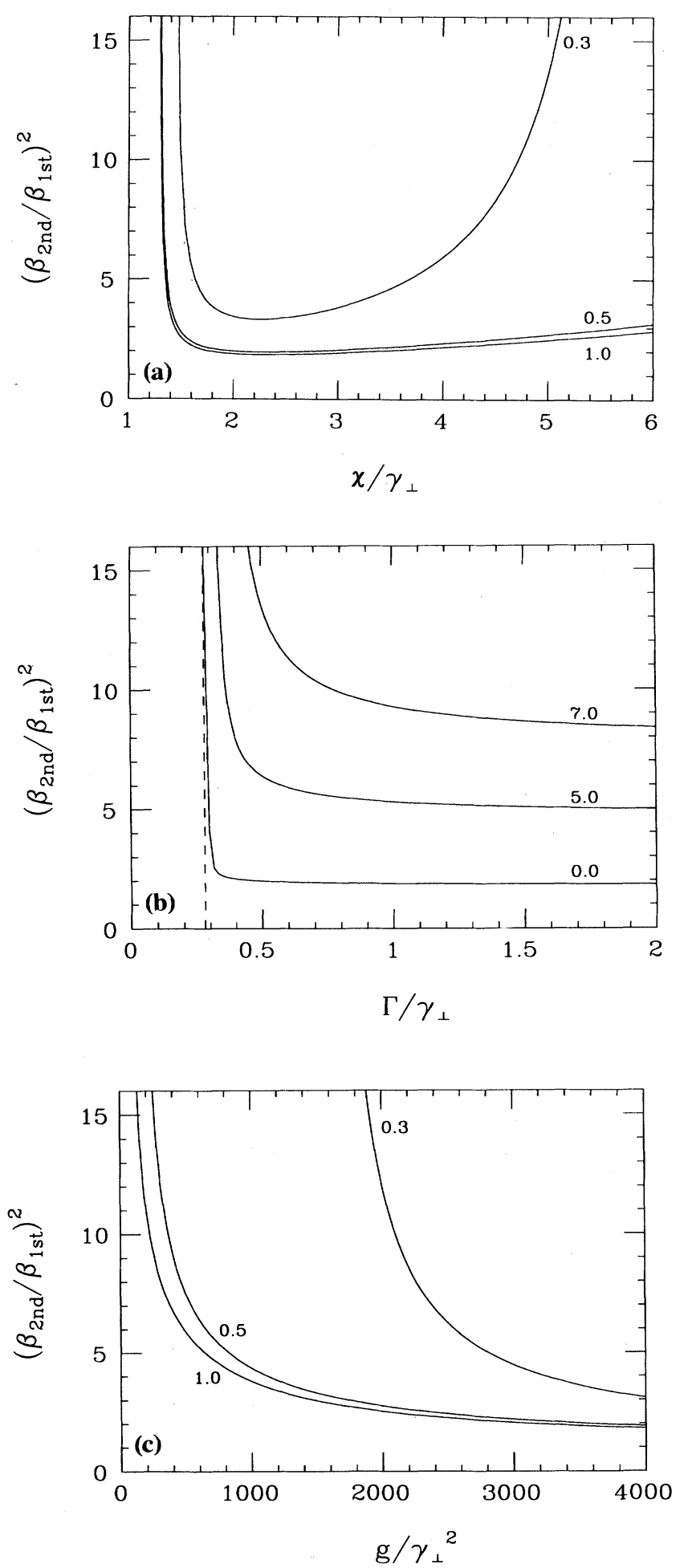

FIG. 11. The same as in Fig. 4, for the truncated model described by Eqs. (13.1), (13.2), and (16).
$\dot{N}_{b c}=\gamma_{\|}\left[\left(\rho_{b b}^{0}-\rho_{c c}^{0}\right)-N_{b c}\right]+6 \alpha y_{c b}-2 \beta y_{b a}$,

$\dot{\rho}_{b a}=-\gamma_{a b} \rho_{b a}+i \beta\left(\rho_{b b}-\rho_{a a}+x_{+-}\right)-i \alpha \rho_{c a}$,

$\dot{\rho}_{c b}=-\left[\gamma_{\perp}+i\left(\Delta_{1}^{c}+\dot{\phi}\right)\right] \rho_{c b}-i \alpha\left(N_{b c}+x_{+-}\right)+i \beta \rho_{c a}$,

$\dot{\rho}_{c a}=-\left[\gamma_{a c}+i\left(\Delta_{1}^{c}+\dot{\phi}\right)\right] \rho_{c a}-2 i \alpha \rho_{b a}+2 i \beta \rho_{c b}$,

$\dot{x}_{+-}=-\Gamma x_{+-}-2 \beta y_{b a}+2 \alpha y_{c b}$,

$\dot{\alpha}=-\kappa \alpha-g y_{c b}$,

$\dot{\phi}=g x_{c b} / \alpha$.

Let us point out that at variance with the case of orthogonal polarizations, in the present case Raman processes and other coherent coupling processes between pump and generated fields governed by the two-photon coherence $\rho_{c a}$ are present. Also since $x_{a b} \neq 0$, there are now dispersive effects at the pump frequency. These fundamental differences between both cases make them dramatically different.

Owing to the complexity of system (21) it is impossible to obtain general analytical results. Moreover, in the few particular cases in which this can be done the obtained expressions are not easy to handle, so we will study only numerically the dynamics of our system.

\section{B. Dynamics}

The phase diagrams of Fig. 12 show in the parameter plane $\left(\beta, \Delta_{1}^{c}\right)$ the different emission regimes of the laser intensity $\alpha^{2}(t)$, obtained by hard-mode excitation. All the dynamic regimes shown here coexist with a steady lasing state, obtained by soft-mode excitation. A comparison of Figs. 5 and 12 shows that the laser is much more stable operating with parallel polarizations than with the orthogonal configuration [31]. In fact, while the instability pump threshold is $\beta=0.0743$ at $\Delta_{1}^{c}=0$ for chaotic emission in Fig. 5(b), it is increased to $\beta=1.54$ at $\Delta_{1}^{c}=0$ for regular period-1 pulsing $\left(P^{1}\right)$ in Fig. 12(b). A global feature of Figs. 5 and 12 is a simplification of the dynamics when the cavity detuning is sufficiently increased. This feature appears also in other laser models. Howev-
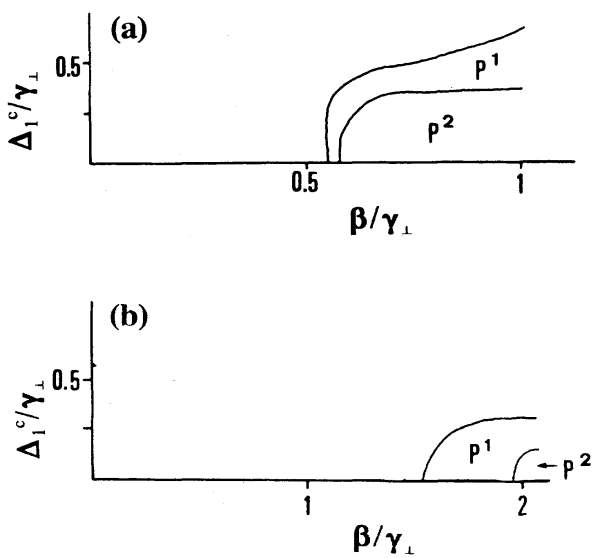

FIG. 12. As in Fig. 5, but for parallel linear polarizations of pump and generated fields. 

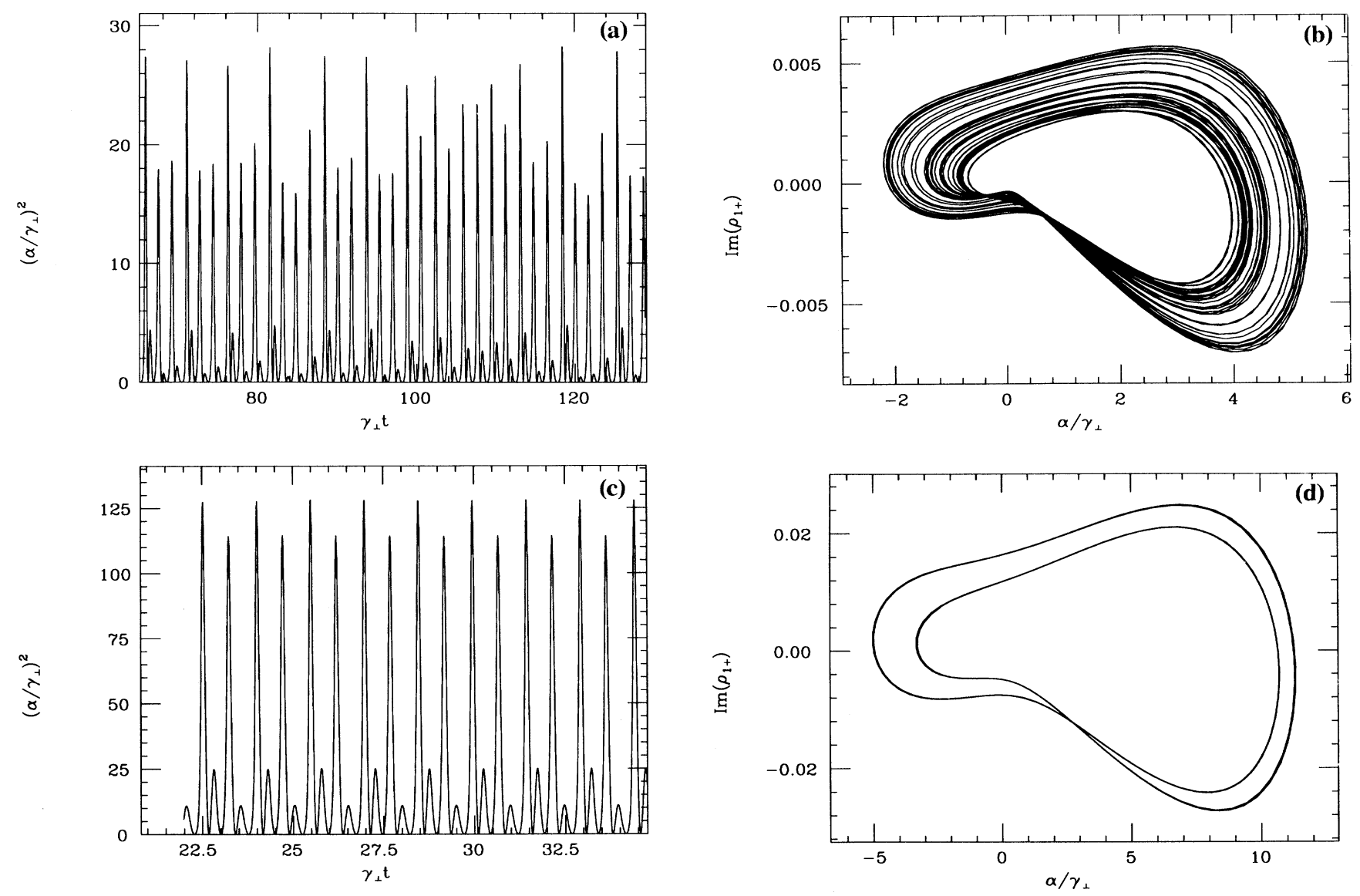

FIG. 13. Intensity pulsing $\alpha^{2}(t)$ and two-dimensional $\left(\alpha, \operatorname{Im} \rho_{c+}\right)$ projections of the dynamic attractor found with hard-mode excitation in the parallel polarizations model for $\Gamma=\gamma_{\|}$and $\Delta_{1}^{c}=0$. The pump field strength in the chaotic regime (a) and (b) is $\beta=0.099 \gamma_{\perp}$; in the period-4 pulsing regime (c) and (d) it is $\beta=0.5 \gamma_{\perp}$ (other parameters as in Fig. 2).

er, our results indicate that the laser with crossed polarizations is less sensitive than the one with parallel polarization to the stabilizing effect of detuning. Note, in fact, that the range of $\Delta_{1}^{c}$ values considered in Fig. 5 is about one order of magnitude larger then in Fig. 12. Note also in Fig. 12 that the instability pump threshold increases by increasing $\Gamma$. This again indicates that the coherence between the upper degenerate levels has a strong influence on the laser dynamics. This point is made clearer in Fig. 13, which corresponds to the dynamic behavior observed for the minimum value of $\Gamma\left(=\gamma_{\|}\right)$for which the parallel configuration laser is most unstable.

While with crossed polarizations the system would not lase for $\Gamma=\gamma_{\|}$, it exhibits now a chaotic pulsing for $\beta=0.099, \Delta_{1}^{c}=0$ [Fig. 13(a)]. Keeping $\Delta_{1}^{c}$ fixed and increasing $\beta$ the chaotic attractor transforms into a periodic one. The period-4 pulsing regime is shown in Fig. 13(c). The chaotic attractor found here does not have the butterfly shape [Fig. 13(b)] characteristic of the Lorenz model but closely resembles the ones found in three-level OPML models [21].

\section{Discussion}

The dynamics just reported is quite different from that found for the orthogonal case. On the other hand, a cer- tain resemblance exists between the results shown in Sec. IV $B$ and those presented in Ref. [21]. This similarity is quite surprising since as was indicated in Sec. II A the model of Ref. [21] corresponds to pump and generated fields with equal circular polarization.

Since the greatest similarity between the parallel case and the circular case [21] occurs when $\Gamma=\gamma_{\|}$let us make this particularization into Eqs. (21). One obtains

$$
\begin{aligned}
& \dot{\sigma}_{b b}=\gamma_{\|}\left(\rho_{b b}^{0}-\sigma_{b b}\right)+4 \alpha y_{c b}-4 \beta y_{b a}, \\
& \dot{D}_{b c}=\gamma_{\|}\left[\left(\rho_{b b}^{0}-\rho_{c c}^{0}\right)-D_{b c}\right]+8 \alpha y_{c b}-4 \beta y_{b a}, \\
& \dot{\rho}_{c b}=-\left[\gamma_{\perp}+i\left(\Delta_{1}^{c}+\dot{\phi}\right)\right] \rho_{c b}-i \alpha D_{b c}+i \beta \rho_{c a}, \\
& \dot{\rho}_{b a}=-\gamma_{b a} \rho_{b a}+i \beta\left(\sigma_{b b}-\rho_{a a}\right)-i \alpha \rho_{c a},
\end{aligned}
$$

which together with Eqs. (21.1), (21.6), (21.8), and (21.9) form a closed set of equations. In Eqs. (22) $\sigma_{b b} \equiv \rho_{b b}+x_{+-}$and $D_{b c}=\sigma_{b b}-\rho_{c c}$.

This closed system is isomorphic to that of Refs. [19] and [21] [38]. This fact, which is in agreement with the numerical results of Sec. IV B, confirms the surprising conclusion that in the limit condition $\Gamma=\gamma_{\|}$, the behavior of the three-level OPML model (circular polarization) is identical to that of the parallel-polarization 
OPML four-level model reported in this section. Thus, all the analytical and numerical results given in Refs. [19] and [21] apply also to the present case. Moreover, the numerical results indicate that the effect of increasing $\Gamma$ is to make the behavior of the four-level system progressively different from that of the three-level model.

\section{CONCLUSIONS AND OUTLOOK}

In this paper we have analyzed in detail the influence of light polarization on the dynamics of an optically pumped single-mode laser with a homogeneously broadened four-level medium. As typical in far-infraredlaser experiments we have considered pump and laser fields with either parallel or crossed linear polarizations.

Dramatically different dynamic behaviors have been found for these two polarization configurations, which suggest that light polarization and level degeneracy are key ingredients of the OPML dynamics. The dynamics strongly depends on the newly introduced relaxation rate $\Gamma$ of the coherence between the two degenerate levels shared by the pump and lasing transitions.

When $\Gamma$ takes its lowest possible value $\left(\Gamma=\gamma_{\|}\right)$, our model for crossed polarizations exactly reduces to the Lorenz-Haken one, while for parallel polarization it is isomorphic with the coherently pumped three-level model. This result enables one to get some insight into the influence the Doppler broadening (not considered in this paper) should have in our four-level model, since the cases of two-level and three-level lasers with Doppler broadening have been previously investigated in Refs. [39] and [22-26], respectively.

In the configuration with crossed polarizations it has been shown that there is no coherent coupling between pump and laser fields for any set of parameter values, at variance with what happens for the three-level model. This allows for the persistence of Lorenz-type behavior over a wide domain of laser parameters. Distinctive features have also been observed, however, that are due to incoherent optical pumping effects. These features include the possibility to have population inversion without lasing; a nonlinear dependence of the steady laser intensity with the pump intensity; the dependence on various laser parameters of the ratio between pump intensities for second and first laser thresholds; or the structure of intensity return maps.

In the parallel-polarization configuration there is coherent coupling between the pump and laser fields and the OPML exhibits a rich and varied dynamics, including periodic and chaotic regimes, very different from the Lorenz-Haken ones, and already extensively studied in the case $\Gamma=\gamma_{\|}[19,21]$. For this configuration an increase of $\Gamma$, by means of phase interrupting collisions for instance, is found to have a strong stabilizing influence on the OPML.

In the light of the above conclusions let us finally emphasize that a completely acceptable theoretical description of the dynamic behavior of real OPML is not yet available. By way of example let us consider the experimental observation by Weiss and co-workers [6] of
Lorenz-type dynamics on the $81.5-\mu \mathrm{m}$ crossedpolarization line of the $\mathrm{NH}_{3}$ laser. The relaxation rates considered in the present work are appropriate for that line, and our four-level model effectively predicts Lorenz-type dynamics in the case of crossed polarizations (although with some restrictions with respect to the values of $\Gamma$ ). The real laser [6], however, is a Dopplerbroadened system, so that Doppler effects should be taken into account. This represents a long numerical task which will be undertaken in subsequent work. Thus, at present we do not know exactly the result of including these effects in the model with crossed polarizations, but since this configuration is effective in decoupling pump and laser fields, one can speculate that Doppler effects should not destroy the Lorenz-like behavior already present in the homogeneous system (on the contrary, they should make the predicted behavior even closer to that observed).

On the other hand, Lorenz-type behavior appears also in the present parallel-polarization four-level model, if we take $\Gamma=\gamma_{\|}$and include Doppler effects, since we then recover the three-level Doppler model of Refs. [22-26]. Thus if the above conjecture is true and this type of behavior occurs for both crossed and parallel polarizations one would come to the conclusion that light polarization does not strongly influence the laser dynamics. Then one should explain the different behavior observed on the $374-\mu \mathrm{m}$ line of the $\mathrm{NH}_{3}$ laser [28,29], which is a Doppler-broadened system with parallel polarizations, on the basis only of the different parameter settings (gain, relaxation rates, wave number, pump detuning, etc.) for these two lines. On the contrary, if Doppler effects destroy the Lorenz-type dynamics in the four-level model with crossed polarizations, then the four-level model would be in contradiction with experiments (predicts the correct behavior for the wrong polarization configuration). Then one should again improve the model considering, e.g., higher $J$ values (as occurs in the experiments). In our opinion all these are very challenging questions that deserve future work.

\section{ACKNOWLEDGMENTS}

This work was supported by the Dirección General de Investigación Científica y Técnica (Spain), Project No. PB89-0319-CO3.

\section{APPENDIX}

In this appendix we prove Eqs. (4.1)-(4.4) that correspond to the case of crossed polarizations

$$
\beta^{+}=-\beta^{-} \equiv \beta \text {. }
$$

Our starting point is the conjecture

$$
\rho_{++}(t)=\rho_{--}(t) \equiv \rho_{b b}(t) .
$$

Equation (A2) is physically feasible since both levels $(+,-)$ are submitted to equal losses $\left(\gamma_{++}=\gamma_{--}\right)$and gain processes $\left(\rho_{++}^{0}=\rho_{--}^{0}\right.$, and pump and laser intensities are equal for the transitions $a \rightarrow \pm$ and $\pm \rightarrow c$, respec- 
tively). Substitution of Eqs. (A1) and (A2) into relation (2.2) gives

$$
\begin{aligned}
& x_{a c}=0, \\
& y_{a c}=\frac{\alpha}{\beta} y_{+-} .
\end{aligned}
$$

On the other hand introduction of Eq. (A1) into Eqs. (3.4) leads to

$$
\begin{aligned}
& x_{a+}=x_{a-} \equiv x_{a b}, \\
& \dot{y}_{a \mu}=-\gamma_{a b} y_{a \mu}+\beta\left(x_{+-}-D_{\mu a}\right)+\alpha x_{a c} .
\end{aligned}
$$

Addition of Eqs. (A6) $(\mu=+,-)$ with the help of Eqs. (A2) and (A3) gives

$$
y_{a+}=-y_{a-} .
$$

Finally, introduction of Eqs. (A1), (A3), (A5), and (A7) into Eqs. (3.6) leads to

$$
\begin{aligned}
& y_{a c}=0, \\
& x_{a b}=0,
\end{aligned}
$$

and substitution of Eq. (A9) into Eq. (A4) gives

$$
y_{+-}=0
$$

Let us point out that conjecture (A2) is consistent with Eqs. (3), although we have not been able to prove its necessity. Nevertheless all the numerical simulations both under stationary and dynamical regimes verify Eq. (A2) and, consequently, the rest of the results derived in this appendix.
[1] See, e.g., Instabilities and Chaos in Quantum Optics, edited by F. T. Arecchi and R. G. Harrison (Springer, Berlin, 1987); N. B. Abraham, P. Mandel, and L. M. Narducci, in Progress in Optics $X X V$, edited by E. Wolf (Elsevier, Amsterdam, 1988), pp. 3-190; C. O. Weiss and R. Vilaseca, Dynamics of Lasers (VCH, Weinheim, 1991).

[2] N. G. Douglas, Millimetre and Submillimetre Wavelength Lasers (Springer, Berlin, 1989).

[3] R. G. Harrison and D. J. Biswas, Phys. Rev. Lett. 55, 63 (1985).

[4] J. S. Uppal, K. Jiad, W. Forysiak, and R. G. Harrison, in International Conference on Quantum Electronics Technical Digest Series 1992, edited by G. Magerl (XVIII International Quantum Electronics Conference, Vienna, 1992), Vol. 9, pp. 300, 301.

[5] C. O. Weiss and W. Klische, Opt. Commun. 51, 47 (1984).

[6] C. O. Weiss and J. Brock, Phys. Rev. Lett. 57, 2804 (1986); U. Hübner, N. B. Abraham, and C. O. Weiss, Phys. Rev. A 40, 6354 (1989).

[7] C. O. Weiss, N. B. Abraham, and U. Hübner, Phys. Rev. Lett. 61, 1587 (1988).

[8] M. Y. Li, Tin Win, C. O. Weiss, and N. R. Heckenberg, Opt. Commun. 80, 119 (1990).

[9] D. Y. Tang, C. O. Weiss, E. Roldán, and G. J. de Valcárcel, Opt. Commun. 89, 47 (1992).

[10] T. Q. Wu, V. Schröder, F. I. Saad, W. Klische, and C. O. Weiss, Infrared Phys. 29, 325 (1989).

[11] H. Haken, Phys. Lett. A 53, 77 (1975); E. Lorenz, J. Atmos. Sci. 10, 130 (1963).

[12] C. T. Sparrow, The Lorenz Equations: Bifurcations, Chaos and Strange Attractors (Springer, Berlin, 1982).

[13] H. Zeghlache and P. Mandel, J. Opt. Soc. Am. B 2, 18 (1985).

[14] D. Y. Tang, M. Y. Li, and C. O. Weiss, Phys. Rev. A 46, 676 (1992)

[15] D. Y. Tang, J. Pujol, and C. O. Weiss, Phys. Rev. A 44, R35 (1991).

[16] M. A. Dupertuis, R. R. E. Salomaa, and M. R. Siegrist, Opt. Commun. 57, 419 (1986).

[17] N. M. Lawandy and J. C. Ryan, Opt. Commun. 63, 53 (1987); J. C. Ryan and N. M. Lawandy, ibid. 64, 54 (1987).
[18] P. A. Khandokhin, Ya. I. Khanin, and I. V. Koryukin, Opt. Commun. 65, 367 (1988).

[19] J. V. Moloney, J. S. Uppal, and R. G. Harrison, Phys. Rev. Lett. 59, 2868 (1987); J. S. Uppal, R. G. Harrison, and J. V. Moloney, Phys. Rev. A 36, 4823 (1987); J. V. Moloney, W. Forysiak, J. S. Uppal, and R. G. Harrison, ibid. 39, 1277 (1989); W. Forysiak, J. V. Moloney, and R. G. Harrison, Physica D 53, 162 (1991).

[20] S. C. Mehendale and R. G. Harrison, Phys. Rev. A 34, 1613 (1986); S. C. Mehendale and R. G. Harrison, Opt. Commun. 60, 257 (1986); J. S. Uppal, R. G. Harrison, and J. V. Moloney, Opt. Commun. 64, 357 (1987); R. G. Harrison and Weiping Lu, Phys. Rev. Lett. 63, 1772 (1989); Weiping Lu and R. G. Harrison, Phys. Rev. A 41, 6563 (1990); Weiping $\mathrm{Lu}$ and R. G. Harrison, ibid. 43, 6358 (1991).

[21] J. Pujol, F. Laguarta, R. Vilaseca, and R. Corbalán, J. Opt. Soc. Am. B 5, 1004 (1988); J. Pujol, F. Laguarta, R. Corbalán, and R. Vilaseca, J. Phys. D 21, S-180 (1988).

[22] F. Laguarta, J. Pujol, R. Vilaseca, and R. Corbalán, J. Phys. (Paris) Colloq. 49, C2-409 (1988).

[23] R. Corbalán, F. Laguarta, J. Pujol, and R. Vilaseca, Opt. Commun. 71, 290 (1989).

[24] E. Roldán, G. J. de Valcárcel, R. Vilaseca, F. Silva, J. Pujol, R. Corbalán, and F. Laguarta, Opt. Commun. 73, 506 (1989).

[25] G. J. de Valcárcel, E. Roldán, and R. Vilaseca, J. Opt. Soc. Am. B 8, 2420 (1991).

[26] C. O. Weiss, R. Vilaseca, N. B. Abraham, R. Corbalán, E. Roldán, G. J. de Valcárcel, J. Pujol, U. Hübner, and D. Y. Tang (unpublished).

[27] V. O. Petukhov, S. Ya. Tochitsky, and V. V. Churakov, Opt. Commun. 72, 87 (1989).

[28] E. H. M. Hogenboom, W. Klische, C. O. Weiss, and A. Godone, Phys. Rev. Lett. 55, 2571 (1985).

[29] M. P. Sassi, N. Barbeau, and C. O. Weiss, Appl. Phys. B 43, 179 (1987).

[30] S. Bielawski, D. Derozier, and P. Glorieux, Phys. Rev. A 46, 2811 (1992).

[31] M. Arjona, R. Corbalán, F. Laguarta, J. Pujol, and R. Vilaseca, Phys. Rev. A 41, 6559 (1990). Let us point out a 
typographical error in the right-hand side of Eq. (2b) in this work. The term $\left[\gamma_{a c}+i\left(\Delta_{1}^{+}-\Delta_{2}^{+}\right)\right] \rho_{a b}$ should read $\left[\gamma_{a c}-i\left(\Delta_{1}^{+}-\Delta_{2}^{+}\right)\right] \rho_{a b}$.

[32] E. Roldán, G. J. de Valcárcel, R. Vilaseca, and R. Corbalán (unpublished).

[33] From now on we find it convenient to scale the time and other variables and parameters to the relaxation rate $\gamma_{\perp}$. Equation (6) and the subsequent formulas in Sec. III are written with this normalization, although we keep the same symbols as before in order to not complicate the notation unnecessarily.

[34] H. Haken, Light (North-Holland, Amsterdam, 1985), Vol. 2.

[35] H. Zeglache, P. Mandel, N. B. Abraham, and C. O. Weiss, Phys. Rev. A 38, 3128 (1988); R. Vilaseca, G. J. de Valcárcel, and E. Roldán, ibid. 41, 5269 (1990).

[36] P. Mandel and O. Kocharovskaya, in Nonlinear Dynamics and Quantum Phenomena in Optical Systems, edited by R. Vilaseca and R. Corbalán (Springer, Berlin, 1991).

[37] (a) H. Zeghlache, Ph.D. thesis, Université Libre de Bruxelles, 1988; (b) P. Mandel and H. Zeghlache, Opt. Commun. 47, 146 (1983); (c) A. Bakasov and N. B. Abraham, ICTP Report No. IC/92/390 (unpublished).

[38] More precisely, our model is isormorphic to that of Ref. [19] only if one takes $\rho_{b b}^{0}=\rho_{c c}^{0}=0, \rho_{a a}^{0}=1$; and is isomorphic to that of Ref. [21] if one takes the population of the upper common level in absence of fields to be half its actual value, maintaining the populations of the other two levels unchanged.

[39] See, e.g., N. B. Abraham, L. A. Lugiato, P. Mandel, L. M. Narducci, and D. K. Bandy, J. Opt. Soc. Am. B 2, 35 (1985); M. F. H. Tarroja, N. B. Abraham, D. K. Bandy, and L. M. Narducci, Phys. Rev. A 34, 3148 (1986); L. W. Casperson, J. Opt. Soc. Am. B 5, 958 (1988); 5, 970 (1988). 